\title{
EXAMINING THE EFFECT OF FEATURE SELECTION ON IMPROVING PATIENT DETERIORATION PREDICTION
}

\author{
Noura AlNuaimi, Mohammad M Masud and Farhan Mohammed \\ College of Information Technology, United Arab Emirates University, Al-Ain, UAE
}

\begin{abstract}
Large amount of heterogeneous medical data is generated every day in various healthcare organizations. Those data could derive insights for improving monitoring and care delivery in the Intensive Care Unit. Conversely, these data presents a challenge in reducing this amount of data without information loss. Dimension reduction is considered the most popular approach for reducing data size and also to reduce noise and redundancies in data. In this paper, we are investigate the effect of the average laboratory test value and number of total laboratory in predicting patient deterioration in the Intensive Care Unit, where we consider laboratory tests as features. Choosing a subset of features would mean choosing the most important lab tests to perform. Thus, our approach uses state-of-the-art feature selection to identify the most discriminative attributes, where we would have a better understanding of patient deterioration problem. If the number of tests can be reduced by identifying the most important tests, then we could also identify the redundant tests. By omitting the redundant tests, observation time could be reduced and early treatment could be provided to avoid the risk. Additionally, unnecessary monetary cost would be avoided. We apply our technique on the publicly available MIMIC-II database and show the effectiveness of the feature selection. We also provide a detailed analysis of the best features identified by our approach.
\end{abstract}

\section{KEYWORDS}

Data mining; patient deterioration; ICU; lab test; feature selection; learning algorithm

\section{INTRODUCTION}

The last decade has seen huge advances in the amount of data that is generated and collected in the modern intensive care units (ICUs), as well as the technologies used to analyse and understand it. ICUs are specialist hospital wards, where they provide intensive care (treatment and monitoring) for patients in seriously ill and their condition changes often. ICUs are considered a critical environment where the decision needs to be carefully taken. These data could be used with the help of intelligent systems, such as data analytics and decision support systems, to determine which patients are at an increased risk of death. Making such decision could allow healthcare professionals to take action at an early stages. For instance, patients in the ICUs have a wide variety of medical laboratory tests on different body fluids (E.g. blood and urine). The natures of medical lab tests and how often these tests are performed depend on why the patient is in ICU and how stable the patient is.

Medical professionals may order laboratory tests to confirm a diagnosis or monitor patients' health. However, deciding which test is likely to contribute information gain is a challenge. DOI : $10.5121 /$ ijdkp.2015.5602 
Recent studies have demonstrated that frequent laboratory testing does not necessarily relate to better outcomes [1].

Dimension reduction would be the first solution to eliminate duplicate, useless and irrelevant features. This is typical alternative done while solving machine learning problems to select the most discriminative attributes. In this paper, our goal is to propose an efficient mining technique to reduce the observation time in ICUs by predicting patient deterioration in its early stages through data analytics. Our proposed technique has several contributions. First, we use the lab test results to predict patient deterioration. To the best of our knowledge, this is the first work that primarily uses medical lab tests to predict patient deterioration. Lab test results have a crucial role in medical decision making. Second, we identify most important medical lab tests using state-ofthe-art feature-selection techniques without using any informed domain knowledge. Finally, our approach helps reduce redundant medical lab tests. Thus, healthcare professionals could focus on the most important lab tests to assist them, which would save not only costs but also valuable time in recovering the patient from a critical condition.

The paper is organised as follows. Section 2 presents the related work of predicting ICU death, Section 3 gives background on data mining, Section 4 illustrates our proposed approach, Section 5 summarises the MIMIC II dataset, Section 6 illustrates the experiment's work, Section 7 discusses the findings, and finally, the conclusion of this research is presented in Section 8.

\section{LITERATURE REVIEW}

This section reviews related works for predicting ICU death or the deterioration of ICU patients, where ICUs workflow is filled with large quantities of data that need more analysis. Most of efforts here are indented to identify redundancy or overlapping between medical laboratory tests. ICUs like any other domain, need regular improvement at their processes and frequent requested medical laboratory tests. In this section, we highlight some similarities and differences between some of the related works and the proposed work.

In [2], the authors developed an integrated data-mining approach to give early deterioration warnings for patients under real-time monitoring in the ICU and real-time data sensing (RDS). They synthesised a large feature set that included first- and second-order time-series features, detrended fluctuation analysis (DFA), spectral analysis, approximative entropy and cross-signal features. Then, they systematically applied and evaluated a series of established data-mining methods, including forward feature selection, linear and nonlinear classification algorithms, and exploratory under sampling for class imbalance. In our work, we are using the same dataset. However, we are using only the medical lab tests. Also, in our approach, we depend on feature selection to reduce the size of the dataset.

A health-data search engine was developed in [3] that supported predictions based on the summarised clusters patient types which claimed that it was better than predictions based on the non-summarised original data. In our work, we use only the medical lab tests, and we attempt to highlight the most important medical labs.

Liu et al. [4] investigated the minimum number of features that was required for a given learning machine to achieve "satisfactory" performance. In their work, an ad hoc heuristic method based on feature-ranking algorithms was used to perform the experiment on six datasets. They found 
that the heuristic method is useful in finding the critical feature dimension for large datasets. In our work, we also use the ranking to rank the most useful features. However, we attempt to investigate the percentage of selected features that would be enough to have moderate model accuracy.

Cismondi et al. [1] proposed reducing unnecessary lab testing in the ICU. Their approach designed on predicting when a proposed future laboratory test may likely to contribute information gain and thereby influence clinical management in patients with gastrointestinal bleeding. At their experiment, there were 11 input variables in total. Ten of these were derived from bedside monitor trends heart rate, oxygen saturation, respiratory rate, temperature, blood pressure, and urine collections, as well as infusion products and transfusions. The final input variable was a previous value from one of the eight laboratory tests being predicted: calcium, PTT, hematocrit, fibrinogen, lactate, platelets, INR and hemoglobin. The outcome for each laboratory test was a binary framework defining whether a laboratory test result contributed information gain or not. Predictive modelling was applied to recognize unnecessary laboratory tests in a real world ICU database extract comprising 746 patients with gastrointestinal bleeding. This work is the closest one to our research; they have the same objective of reducing unnecessary laboratory tests. However, they only focus on gastrointestinal bleeding. In our work, we are targeting all cases in the ICUs. Besides that, they had constraints on the medical laboratory tests, where they specify eight laboratory tests to be predicted.

Similarly Joon Lee and David M. Maslove [5] used information theory to identify the unnecessary laboratory testing and bloodwork. They investigated the information content of 11 laboratory test results from 29,149 adult ICU admissions in the MIMIC II database. They used Information theory to count the expected amount of redundant information both between laboratory values from the same ICU day, and between consecutive ICU days. They found out that most laboratory values showed a decreasing trend over time in the expected amount of novel information they contained. Platelet, blood urea nitrogen (BUN), and creatinine measurements exhibited the most amount of redundant information on days 2 and 3 compared to the previous day. The creatinine-BUN and sodium-chloride pairs had the most redundancy. In our work, we are not investigating any specific laboratory values, but we aim to identify the most critical laboratory tests that need more attention. Also, in our case we are not depending on any domain knowledge and without any intervention from medical experts.

Likewise the previous works, Hsieh et al. [6] worked on reducing unnecessary laboratory tests in the ICUs. They proposed a computational-intelligence-based model to predict the survival rate of critically ill patients who were admitted to an intensive care unit (ICU). At their research, the prediction input variables were based on the first 24 hours admission physiological data of ICU patients to forecast whether the final outcome was survival or not. Their prediction model was based on a particle swarm optimization (PSO)-based Fuzzy Hyper-Rectangular Composite Neural Network (PFHRCNN) that integrated three computational intelligence tools including hyper-rectangular composite neural networks, fuzzy systems and PSO. In our work, we design our experiment on state-of-the-art feature-selection techniques, where no constraint in the input variables. 


\section{DATA Mining BACKGROUND}

ICUs, like other healthcare sectors, is facing the need for analysing large amounts of data. Data mining represents great potential benefits for the ICUs to enable systematically use data and analytics to identify best practices that improve care and reduce costs. Clinical data mining is the application of data mining techniques using clinical data. Data mining with clinical data has three objectives: understanding the clinical data, assist healthcare professionals, and develop a data analysis methodology suitable for medical data [7].

Data mining is the analysis step of knowledge discovery. It is about the "extraction of interesting (non-trivial, implicit, previously unknown, and potentially useful) patterns or knowledge from huge amount of data [10]'. When mining massive datasets, two of the most common, important and immediate problems are sampling and feature selection. Appropriate sampling and feature selection contribute to reducing the size of the dataset while obtaining satisfactory results in model building [4].

\subsection{Feature Selection}

In machine learning, feature selection or attribute selection is the process of selecting a subset of relevant features (variables, predictors) for use in model construction. Feature selection techniques are used (a) to avoid overfitting and improve model performance, i.e. predict performance in the case of supervised classification and better cluster detection in the case of clustering, (b) to provide faster and more cost-effective models and (c) to gain deeper insight into the underlying processes that generated the data. In the context of classification, feature selection techniques can be organized into three categories, depending on how they perform the feature selection search to build the classification model: filter methods, wrapper methods and embedded methods, presented in table 1 [8] [9]:

1) Filter Methods are based on applying a statistical measure to assign a scoring to each feature. Then, features are ranked by score and either selected or removed from the dataset. The methods are often univariate and consider the feature independently or with regard to the dependent variable.

2) Wrapper Methods are based on the selection of a set of features as a search problem, where different combinations are prepared, evaluated and compared to other combinations. A predictive model is used to evaluate a combination of features and assign a score based on model accuracy.

3) Embedded Methods are based on learning which features most contribute to the accuracy of the model while the model is being created.

Table 1: Feature selection categories

\begin{tabular}{|l|l|l|}
\hline Model Search & Advantages & Disadvantages \\
\hline Filter & $\begin{array}{l}\text { Fast } \\
\text { Scalable } \\
\text { Independent of the classifier }\end{array}$ & $\begin{array}{l}\text { Ignores feature dependencies } \\
\text { Ignores interaction with the classifier }\end{array}$ \\
\hline Wrapper & $\begin{array}{l}\text { Simple } \\
\text { Interacts with the classifier } \\
\text { Models feature decencies } \\
\text { Less computational }\end{array}$ & $\begin{array}{l}\text { Risk for overfitting } \\
\text { More prone than randomized algorithms } \\
\text { Classifier-dependent selection }\end{array}$ \\
\hline
\end{tabular}


International Journal of Data Mining \& Knowledge Management Process (IJDKP) Vol.5, No.6, November 2015

\begin{tabular}{|l|l|l|}
\hline Embedded & $\begin{array}{l}\text { Interacts with the classifier } \\
\text { More computational } \\
\text { Models feature dependencies }\end{array}$ & Classifier-dependent selection \\
\hline
\end{tabular}

\subsection{Data Classification Techniques}

Classification is a pattern-recognition task that has applications in a broad range of fields. It requires the construction of a model that approximates the relationship between input features and output categories [10]. Some of the most popular techniques are discussed here in brief, all of which are used in our work.

1) The Naïve Bayes classifier is based on applying Bayes' theorem with strong independence assumptions between the features. As one of its main features, the Naïve Bayes classifier is easy to implement because it requires a small amount of training data in order to estimate the parameters, and good results can be found in most cases. However, it has class conditional independence, meaning it causes losses of accuracy and dependency [11].

2) Sequential minimal optimization (SMO) is an algorithm for efficiently solving the optimization problem which arises during the training of support vector machines [12]. The amount of memory required for SMO is linear in the training set size, which allows SMO to handle very large training sets [13].

3) The ZeroR classifier simply predicts the majority category, which relies on the target and ignores all predictors. Although there is no predictability power in ZeroR, it is useful for determining a baseline performance as a benchmark for other classification methods [12].

4) A decision tree (J48) is a fast algorithm to train and generally gives good results. Its output is human readable, therefore one can see if it makes sense. It has tree visualizers to aid understanding. It is among the most used data mining algorithms. The decision tree partitions the input space of a data set into mutually exclusive regions, each of which is assigned a label, a value or an action to characterize its data points [12].

5) A RandomForest is a combination of tree predictors such that each tree depends on the values of a random vector sampled independently and with the same distribution for all trees in the forest [14].

\section{PROPOSED APPROACH}

In this section we introduce our approach for the data mining technique for predicting ICU patient deterioration. Figure 1 shows the architecture of the proposed technique.

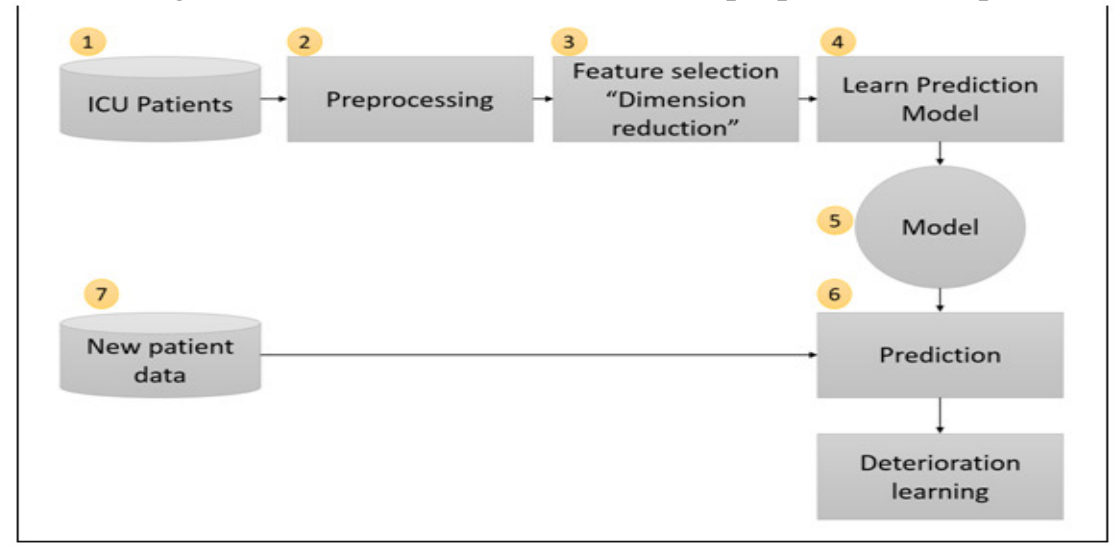

Figure 1: Architecture of the proposed approach. 
The data are collected from the database of ICU patients (step 1). Then the data are integrated, cleaned and relevant features are extracted (step 2). After that, feature selection or dimensionality reduction techniques are applied to obtain the best set of features and reduce the data dimension (step 3). Then the prediction model is learned using a machine learning approach (step 4). When a new patient is admitted to the CPU, the patient's data are collected incrementally (step 5). The patient data are evaluated by the prediction model (step 6) to predict the possibility of deterioration of the patient, and warnings are generated accordingly. Each of these steps is summarized here, and more details of the dataset are given in Section 5.

1. ICU Patient Data: The details of the data and the collection process are discussed in Section 5.

2. Preprocessing: At the preprocessing stage, we used two different datasets. These datasets were generated from a Labevents table. The first dataset contained the average value of applied medical tests, and the second contained the total number of times for each test was applied.

3. Feature Selection / Dimension Reduction: attribute selection is the process of selecting a subset of relevant features (variables, predictors) for use in model construction. The goal here is to reduce the attributes so medical professional can identify the most important medical lab tests used by reducing the redundant tests. In our work, we select filter methods because they are moderately robust against the overfitting problem, as follows:

a. Attribute evaluator: InfoGrainAttributeEval

b. Search method: Ranker

c. Attribute selection mode: use full training set

4. Learning: In our experiment we use a classification technique and five of the most popular classifier techniques: Naïve Bayes classifier, Support vector machine (SVM), ZeroR classifier, decision tree (J48) and RandomForest. We use different types of machine learning order to avoid random results.

5. Model: The developed model aims to predict ICU patient deterioration by mining lab test results. Thus, observation time can be reduced in the ICUs and more actions can be taken in the early stages.

6. Prediction: After each new test result, medication event, etc., the patient data are preprocessed and features are extracted to supply to the prediction model. The model predicts the probability of deterioration for the patient. This probability may change when new data (e.g. more test results) are accumulated and applied to the model. When the deterioration probability reaches a certain threshold specified by the healthcare providers, a warning is generated. This would help the healthcare providers to take proactive measures to save the patient from getting into a critical or fatal condition.

7. New patient data: When a new patient is admitted to the ICU, all his information is stored in the database. Some of these are incremental, such as vital sign readings, lab test results, medication events etc. The data of the patient again go through the preprocessing and feature extraction phases before they can be applied to the model.

\section{MIMIC II DATABASE}

The MIMIC-II database is part of the Multiparameter Intelligent Monitoring in Intensive Care project funded by the National Institute of Biomedical Imaging and Bioengineering at the Laboratory of Computational Physiology at MIT, which was collected from 2001 to 2008 and represents 26,870 adult hospital admissions. In our work, we use MIMIC-II version 2.6 because is more stable than the newer version 3, which is still in the beta phase and needs further work of 
cleaning, optimizing and testing. MIMIC-II consists of two major components: clinical data and physiological waveforms.

The MIMIC dataset has three main features: (1) it is public; (2) it has a diverse and very large population of ICU patients; and (3) it contains high temporal resolution data, including lab results, electronic documentation, and bedside monitor trends and waveforms[15]. Several works have used the MIMIC dataset, such as [16], [17] and [18].

In our work, we focus on the clinical data, the LABEVENTS and LABITEMS tables. The Labevents table contains data of each patient's ICU stay, as presented in table 2, and table 3 contains descriptions of the lab events. Considering medical lab choice was done because we wanted to investigate the relationship between medical lab tests and patient deterioration so we could identify which medical tests have a major effect on clinical decision making. For example, the following information is about a patient who was staying at the ICU and was given a medical test. The following information was recorded at that time:

- Subject_ID: 2

- Hadm_ID: 25967

- IcuStay_ID: 3

- ItemID: 50468

- Charttime: 6/15/2806 21:48

- Value: 0.1

- ValueNum: 0.1

- Flag: abnormal

- ValueUOM: K/uL

Table 2: Labevents Table Description

\begin{tabular}{|c|c|c|c|}
\hline Name & Type & Null & Comment \\
\hline SUBJECT_ID & NUMBER(7) & $\mathrm{N}$ & $\begin{array}{l}\text { Foreign key, referring to a unique patient } \\
\text { identifier }\end{array}$ \\
\hline HADM_ID & NUMBER(7) & $\mathrm{Y}$ & $\begin{array}{l}\text { Foreign key, referring to the hospital } \\
\text { admission ID of the patient }\end{array}$ \\
\hline ICUSTAY_ID & NUMBER(7) & $\mathrm{Y}$ & ICU stay ID \\
\hline ITEMID & NUMBER(7) & $\mathrm{N}$ & $\begin{array}{l}\text { Foreign key, referring to an identifier for the } \\
\text { laboratory test name }\end{array}$ \\
\hline CHARTTIME & $\begin{array}{l}\text { TIMESTAMP(6) WITH } \\
\text { TIME ZONE }\end{array}$ & $\mathrm{N}$ & The date and time of the test \\
\hline VALUE & VARCHAR2(100) & $\mathrm{Y}$ & The result value of the laboratory test \\
\hline VALUENUM & NUMBER(38) & $\mathrm{Y}$ & $\begin{array}{l}\text { The numeric representation of the laboratory } \\
\text { test if the result was numeric }\end{array}$ \\
\hline FLAG & VARCHAR2(10) & $\mathrm{Y}$ & $\begin{array}{l}\text { Flag or annotation on the lab result to } \\
\text { compare the lab result with the previous } \\
\text { or next result }\end{array}$ \\
\hline VALUEUOM & VARCHAR2(10) & $\mathrm{Y}$ & $\begin{array}{l}\text { The units of measurement for the lab result } \\
\text { value }\end{array}$ \\
\hline
\end{tabular}


Table 3: Labitems Table

\begin{tabular}{|l|l|l|l|}
\hline Name & Type & Null & Comment \\
\hline ITEMID & NUMBER(7) & $\mathrm{N}$ & Table record unique identifier, the lab item ID \\
\hline TEST_NAME & VARCHAR2(50) & $\mathrm{N}$ & The name of the lab test performed \\
\hline FLUID & VARCHAR2(50) & $\mathrm{N}$ & The fluid on which the test was performed \\
\hline CATEGORY & VARCHAR2(50) & $\mathrm{N}$ & Item category \\
\hline LOINC_CODE & VARCHAR2(7) & $\mathrm{Y}$ & LOINC code for lab item \\
\hline $\begin{array}{c}\text { LOINC_DESCR } \\
\text { IPTION }\end{array}$ & VARCHAR2(100) & $\mathrm{Y}$ & LOINC description for lab item \\
\hline
\end{tabular}

\subsection{Medical Lab Tests Average Dataset}

The dataset was constructed by taking the average test result of each patient for each kind of test and make it one attribute. Thus one patient would be represented as one instance having 700 attributes, one for each test. If a test was not done, then the value of that attribute would be 0 . For example, the first patient record in the dataset would look like this:

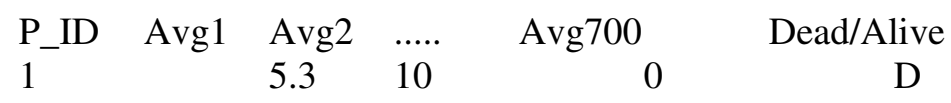

\subsection{Total Number of Medical Lab Tests Dataset}

The dataset was built by taking the total number of tests taken for each patient for each type of test and make it one attribute. Then one patient would be represented as one instance having 700 attributes, one for each test. If a test was not done, then the value of that attribute would be 0 . For example, the dataset would look like this:

\begin{tabular}{lclll} 
P_ID & Count 1 Count $2 \ldots$ & Count700 & \multicolumn{2}{l}{ Dead/Alive } \\
1 & 5 & 0 & 1 & D
\end{tabular}

\section{EXPERIMENTS}

In the experiment section we investigate the effect of feature selection in improving the prediction of patient deterioration in the ICUs. We consider the lab tests as features. Thus, choosing a subset of features would mean choosing the most important lab tests to perform. If the number of tests can be reduced by identifying the most important tests, then we would also identify the redundant tests.

\subsection{Experiment 1: Building a Baseline of the Medical Lab Tests Average}

1) Experiment Goal: The goal of this experiment was to investigate the effect of lab testing on predicting patient deterioration. Usually, medical professionals compare the result of the lab test with a reference range [19]. If the value is not within this range, the patient may face fatal consequences. Thus, the patient is kept under observation and the test is repeated again during a specific period. In our experiment, we investigated the average value of the same repeated test and, more precisely, how the average value of lab results could assist medical professionals in evaluating patient status.

Since we dealt with real cases, the only way to assess the quality and characteristics of a data mining model was through the final status of the patient, i.e. whether the patient survived or 
not. Thus, our evaluation criterion was how accurately our approach could predict whether the patient died or not.

2) Building the Dataset: The dataset was constructed by taking the average test result of each patient for each kind of test and make it one attribute. Thus one patient would be represented as one instance having 700 attributes, one for each test. If a test was not done, then the value of that attribute would be 0 .

For example, the first patient record in the dataset would look like this:

$\begin{array}{llllll}\text { P_ID } & \text { Avg1 } & \text { Avg2 } & \ldots . . & \text { Avg700 } & \text { Dead/Alive } \\ 1 & 5.3 & 10 & & 0 & \text { D }\end{array}$

3) Pre-processing: After building the dataset, some values could not be reported because they were in text format. We used default values for these types of data. The total number of attributes was 619 with 2900 instances.

4) Base learners: In our experiment we used five classification algorithms to construct the model, namely NaiveBayes, SMO, ZeroR, J48 and RandomForest.

5) Evaluation: For a performance measurement, we did a 10-fold cross-validation of the dataset, and the confusion matrix was obtained to estimate four measures: accuracy, sensitivity, specificity and F-measure. As a result, RandomForest had the highest accuracy of $77.58 \%$, followed by SMO with $76.86 \%$, J48 with $75.27 \%$, ZeroR with $70.24 \%$ and NavieBayes with $42.96 \%$, as shown in Table 4, Figure 2 and Figure 3. RandomForest and SMO have the same F-measures. The reason for the best performance by RandomForest is that it works relatively well when used with high-dimensional data with a redundant/noisy set of features [14].

Table 4: Experiment 1confusion matrix results.

\begin{tabular}{|c|c|c|c|c|c|}
\hline \multirow[b]{2}{*}{ Algorithm } & \multirow[b]{2}{*}{ Learning Machine } & \multicolumn{4}{|c|}{ Detailed Accuracy } \\
\hline & & 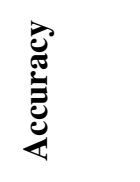 & 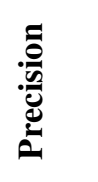 & 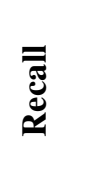 & 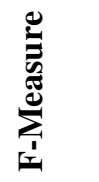 \\
\hline Bayes & NavieBayes & $42.96 \%$ & 0.672 & 0.430 & 0.404 \\
\hline Functions & SMO & $76.86 \%$ & 0.759 & 0.769 & 0.762 \\
\hline Rule & ZeroR & $70.24 \%$ & 0.493 & 0.702 & 0.580 \\
\hline Tree & J48 & $75.27 \%$ & 0.749 & 0.753 & 0.751 \\
\hline Tree & RandomForest & $77.58 \%$ & 0.765 & 0.776 & 0.762 \\
\hline
\end{tabular}

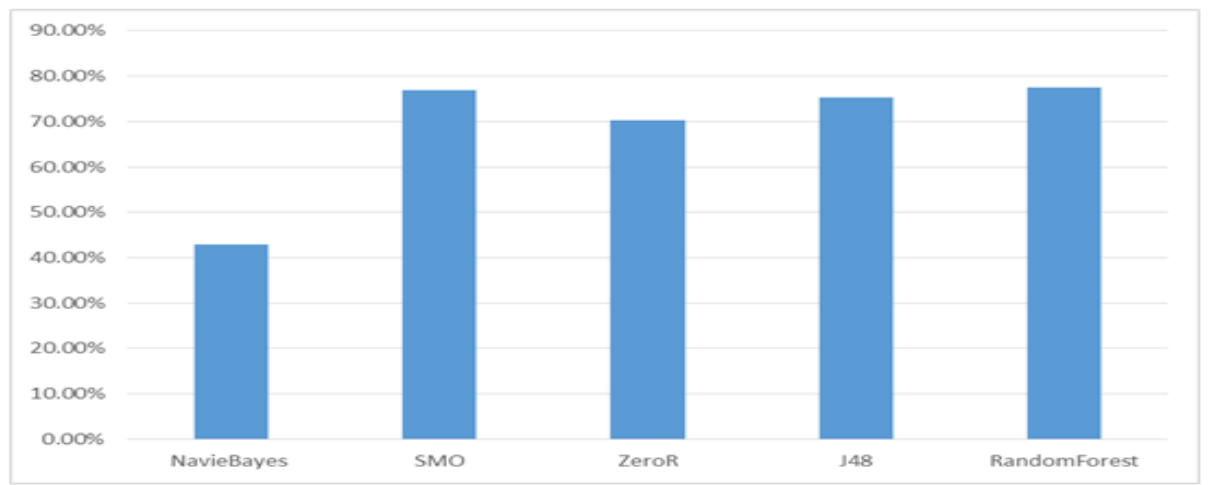

Figure 2: Experiment 1 accuracy result. 


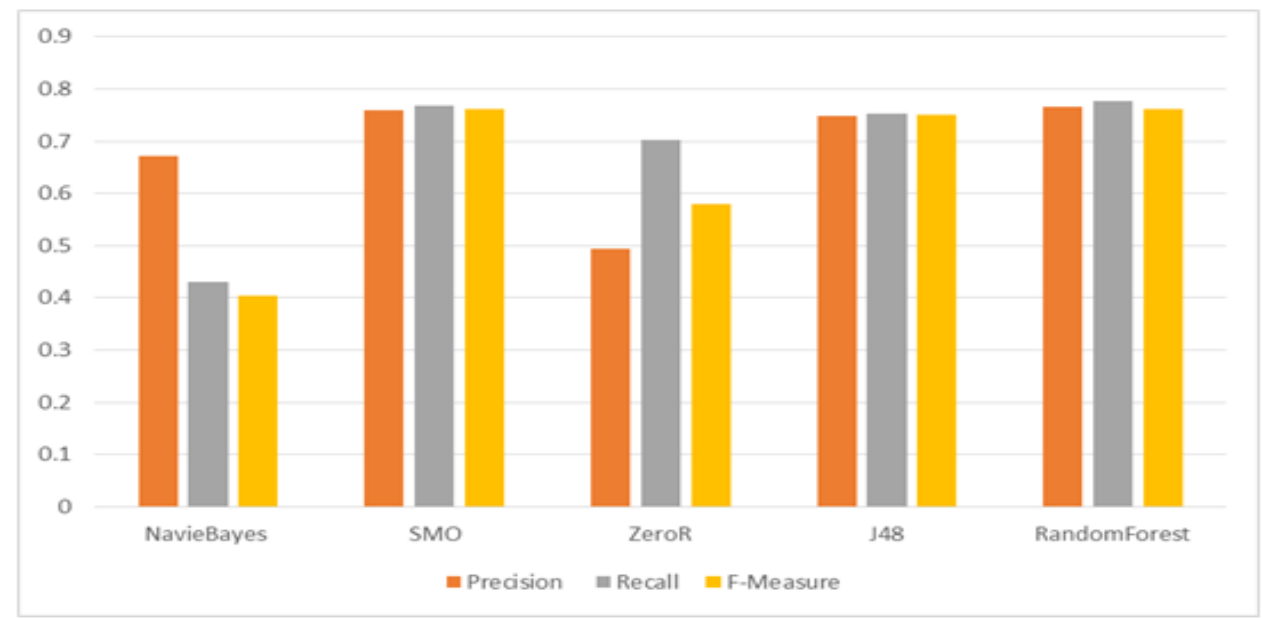

Figure 3: Experiment 1 detailed accuracy result.

\subsection{Experiment 2: Average Medical Lab Tests Discriminative Attributes}

1) Experiment Goal: The goal of this experiment was to select the most discriminative attributes that can almost describe the model with less number of attributes. In this experiment we were working to get the most out of the average medical lab tests data, so we would have a better understanding to patient deterioration problem.

2) Building the Dataset: In this experiment we used the same dataset that we used in experiment 1.

3) Pre-processing: In this stage, we used feature selection to select the most discriminative attributes. For feature selection, we used weka.attributeSelection.CfsSubsetEval from WEKA [20].

- Attribute Subset Evaluator: CfsSubsetEval

- Search Method: BestFirst.

- Evaluation mode: evaluate all training data

4) Base learner: Applying CfsSubsetEval reduced the attributes to 26 selected attributes. Now the goal was to compare the reduced dataset with the baseline experiment result. We used the same five classification algorithms to construct the model, namely NaiveBayes, SMO, ZeroR, J48 and RandomForest. Please refer to Table 5.

Table 5: Experiment 2 confusion matrix result.

\begin{tabular}{|c|c|c|c|c|c|}
\hline \multirow[b]{2}{*}{ Algorithm } & \multirow[b]{2}{*}{ Learning Machine } & \multicolumn{4}{|c|}{ Detailed Accuracy } \\
\hline & & 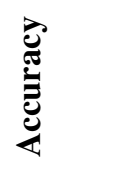 & 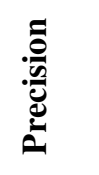 & 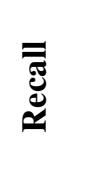 & 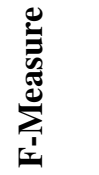 \\
\hline Bayes & NavieBayes & $56.24 \%$ & 0.774 & 0.562 & 0.564 \\
\hline Functions & SMO & $74.82 \%$ & 0.732 & 0.748 & 0.717 \\
\hline Rule & ZeroR & $70.24 \%$ & 0.493 & 0.702 & 0.580 \\
\hline Tree & $\mathrm{J} 48$ & $76.75 \%$ & 0.765 & 0.768 & 0.766 \\
\hline Tree & RandomForest & $79.75 \%$ & 0.790 & 0.798 & 0.789 \\
\hline
\end{tabular}


5) Evaluation: Comparing the accuracy results from this experiment and the first experiment was reported in table. As a result, RandomForest accuracy had the most significant increase, where it increased by $13 \%$. J48 and RandomForest increased had improved slightly. However, SMO and ZeroR did not have any enhancement at their accuracy result. Please refer to Table 6 and Figure 4.

Table 6: Accuracy comparison between Experiment 1 \& Experiment 2.

\begin{tabular}{|l|l|l|l|r|}
\hline Algorithm & $\begin{array}{c}\text { Learning } \\
\text { Machine }\end{array}$ & $\begin{array}{c}\text { Accuracy of } \\
\text { the original } \\
\text { average } \\
\text { dataset }\end{array}$ & $\begin{array}{c}\text { Accuracy of the } \\
\text { reduced average } \\
\text { dataset }\end{array}$ & Change \\
\hline Bayes & NavieBayes & $42.96 \%$ & $56.24 \%$ & \\
\hline Functions & SMO & $76.86 \%$ & $74.82 \%$ & $13.28 \%$ \\
\hline Rule & ZeroR & $70.24 \%$ & $70.24 \%$ & $-2.04 \%$ \\
\hline Tree & J48 & $75.27 \%$ & $76.75 \%$ & $0.00 \%$ \\
\hline Tree & RandomForest & $77.58 \%$ & $79.75 \%$ & $2.17 \%$ \\
\hline
\end{tabular}

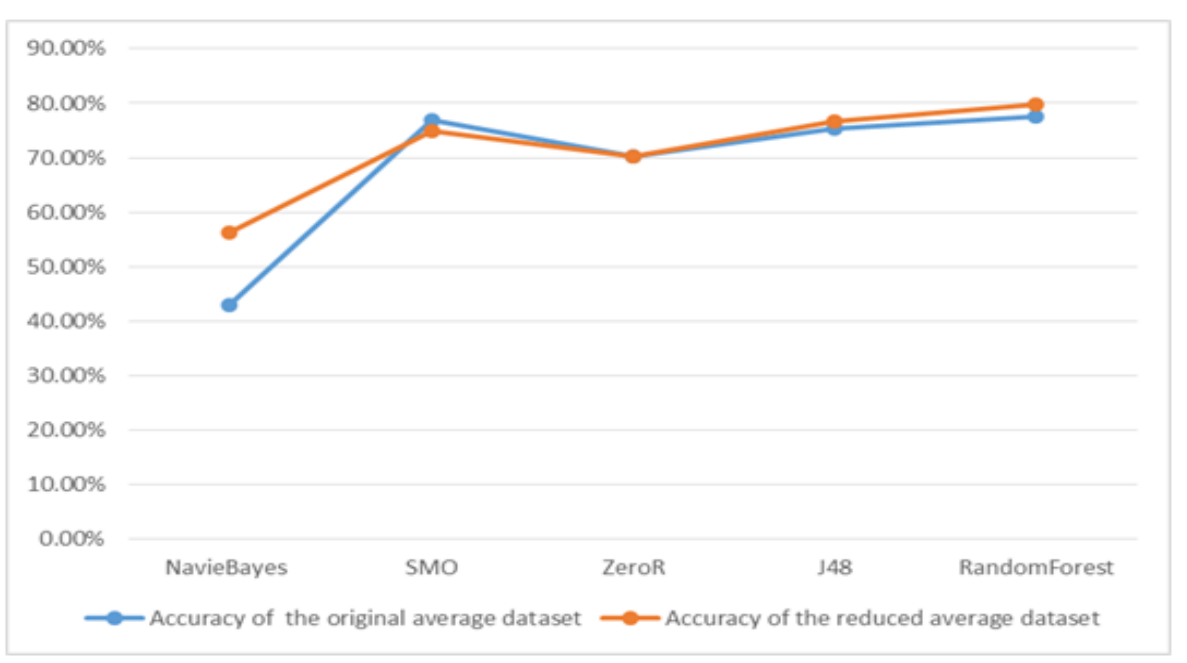

Figure 4: Accuracy comparison between Experiment 1 \& Experiment 2.

\subsection{Experiment 3: Average Medical Lab Tests Feature Selection}

1) Experiment Goal: The goal of this experiment was to study the relationship between feature selection and classification accuracy. Feature selection is one of the dimensionality reduction techniques for reducing the attribute space of a feature set. More precisely, it determines how many features should be enough to give moderate accuracy.

2) Building the Dataset: In this experiment we used the same dataset that we used in experiment 1 .

3) Pre-processing: In this experiment we built ten datasets depending on the number of selected features. We start with the first dataset, which contained only $10 \%$ of the total attributes. Then each time, we increased the total feature selections by $10 \%$. For example, 
dataset 1 contains $10 \%$ of the total attributes, dataset 2 contains $20 \%$ of the total attributes, dataset 3 contains $30 \%$ of the total attributes and so on till dataset 10 contains all $100 \%$ of the total attributes.

For feature selection, we use supervised.attribute. InfoGainAttributeEval from WEKA. This filter is a wrapper for the Weka class that computes the information gain on a class [20].

- Attribute Subset Evaluator: InfoGainAttributeEval

- Search Method: Ranker.

- Evaluation mode: evaluate all training data

4) Base learner: After generating all of the reduced datasets, we used the J48 algorithm to construct a model.

5) Evaluation: For each reduced dataset, we applied 10-fold cross-validation for evaluating the accuracy. Table V shows the results in numbers, and Figure 2 shows them as a chart. The results indicate that taking only the most related $10 \%$ of the total features can give a $75.10 \%$ accurate result, which is comparable to the accuracy of the full feature set. This indicates that not all of the features are required to get the highest accuracy. However, there are some fluctuations, such as at $20 \%$, the accuracy drops a little. We conclude that selecting 50 to $80 \%$ of the attributes should give moderately satisfying accuracy.

Table 7: Experiment 3 feature selection result.

\begin{tabular}{|c|c|c|c|c|}
\hline \multirow[b]{2}{*}{$\%$ of Features Selected } & \multirow[b]{2}{*}{ \# of Features Selected } & \multicolumn{3}{|c|}{ J48 Detailed Accuracy } \\
\hline & & 冚 & 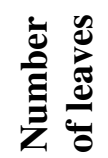 & 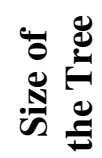 \\
\hline $10 \%$ & 62 & $75.10 \%$ & 200 & 399 \\
\hline $20 \%$ & 124 & $73.59 \%$ & 201 & 401 \\
\hline $30 \%$ & 186 & $75.10 \%$ & 185 & 369 \\
\hline $40 \%$ & 248 & $74.93 \%$ & 179 & 357 \\
\hline $50 \%$ & 310 & $75.17 \%$ & 189 & 377 \\
\hline $60 \%$ & 371 & $74.79 \%$ & 187 & 373 \\
\hline $70 \%$ & 433 & $75.00 \%$ & 189 & 377 \\
\hline $80 \%$ & 495 & $75.31 \%$ & 184 & 367 \\
\hline $90 \%$ & 557 & $74.97 \%$ & 183 & 365 \\
\hline $100 \%$ & 619 & $74.86 \%$ & 184 & 367 \\
\hline
\end{tabular}

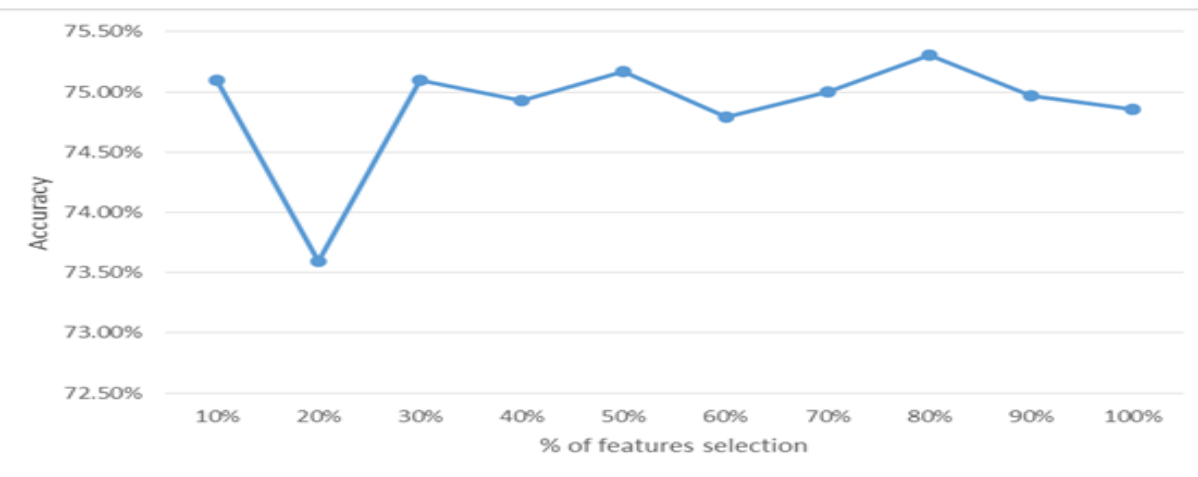

Figure 5: Average datasets accuracy. 


\subsection{Experiment 4: Building a Baseline for the Total Number of Medical Lab Tests}

1) Experiment Goal: The goal of this experiment was to investigate the effect of the total number of lab tests conducted on predicting patient deterioration. Usually, medical professionals keep requesting the same medical test over a brief period to compare the result with a reference range [19]. If the value is not within the range, it means the patient may be in danger, so the test is repeated again and again. Our goal was to predict at what total number a medical professional should start immediate action and, more precisely, how the total number of medical lab tests could assist the medical professional in evaluating the patient's status.

2) Building the Dataset: The dataset was built by taking the total number of tests taken for each patient for each type of test and make it one attribute. Then one patient would be represented as one instance having 700 attributes, one for each test. If a test was not done, then the value of that attribute would be 0 .

For example, the dataset would look like this:

$\begin{array}{llcll}\text { P_ID } & \text { Count1 Count2 } & \ldots & \text { Count700 } & \text { Dead/Alive } \\ 1 & 5 & 0 & 1 & \text { D }\end{array}$

3) Pre-processing: The dataset was randomized first, then two datasets were generated, Count_Training_Validation_Dataset and Count_testing_Dataset. This step was repeated ten times because we used randomization to distribute the instances between the two datasets.

4) Base learners: Five learning algorithms were used to build the model, namely NaiveBayes, SMO, ZeroR, J48 and RandomForest.

Table 8: Experiment 4 confusion matrix Results.

\begin{tabular}{|c|c|c|c|c|c|}
\hline \multirow[b]{2}{*}{ Algorithm } & \multirow[b]{2}{*}{ Learning Machine } & \multicolumn{4}{|c|}{ Detailed Accuracy } \\
\hline & & 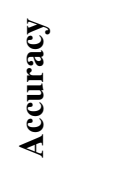 & 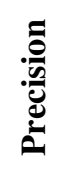 & 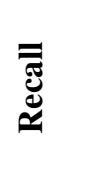 & 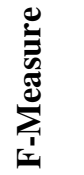 \\
\hline Bayes & NavieBayes & $73.48 \%$ & 0.716 & 0.735 & 0.711 \\
\hline Funtions & SMO & $74.85 \%$ & 0.737 & 0.749 & 0.716 \\
\hline Rule & ZeroR & $69.72 \%$ & 0.486 & 0.697 & 0.573 \\
\hline Tree & $\mathrm{J} 48$ & $72.44 \%$ & 0.722 & 0.724 & 0.723 \\
\hline Tree & RandomForest & $75.30 \%$ & 0.739 & 0.753 & 0.736 \\
\hline
\end{tabular}

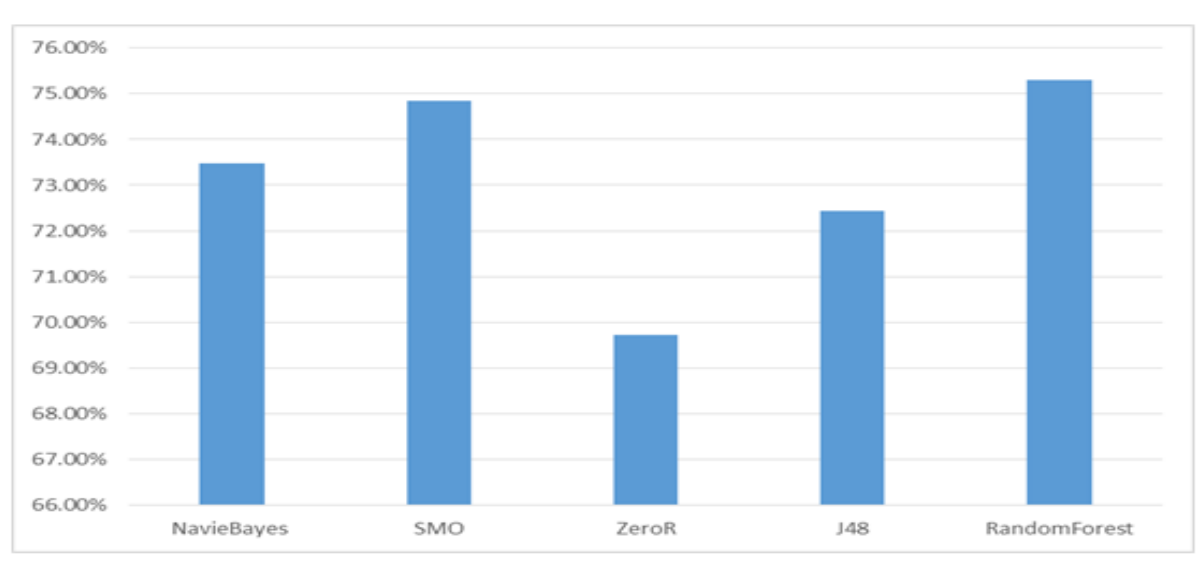

Figure 6: Experiment 4 accuracy result. 


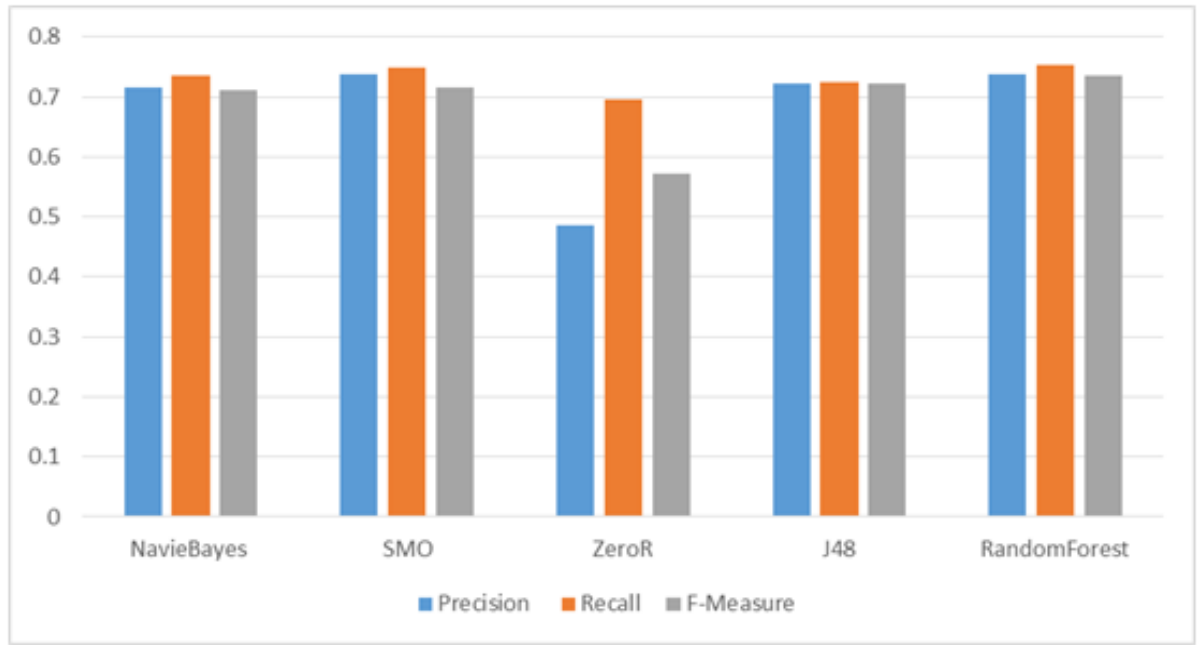

Figure 6: Experiment 4 detailed accuracy result.

5) Evaluation: The training data were first used to build the model and then evaluated using a percentage split via test data. For a performance measurement, the confusion matrix was obtained to estimate four measures: accuracy, sensitivity, specificity and F-measure. Table 6 shows that SMO and RandomForest have almost equal levels of accuracy, around $75 \%$. Even after testing the model with the test datasets, SMO and RandomForest still have the highest accuracy among the other techniques. The reason for this higher accuracy is that the amount of memory required for SMO is linear in the training set size, which allows SMO to handle very large training sets [13].

\subsection{Experiment 5: Total Number of Medical Lab Tests Discriminative Attributes}

1) Experiment Goal: The goal of this experiment was to select the most discriminative attributes that can almost describe the model with less number of attributes. In this experiment we were working to get the most out of the total number of medical lab tests data, so we would have a better understanding to patient deterioration problem.

2) Building the Dataset: In this experiment we used the same dataset that we used in experiment 4.

3) Pre-processing: In this stage, we used feature selection to select the most discriminative attributes. For feature selection, we used weka.attributeSelection.CfsSubsetEval from WEKA [20].

- Attribute Subset Evaluator: CfsSubsetEval

- Search Method: BestFirst.

- Evaluation mode: evaluate all training data

4) Base learner: Applying CfsSubsetEval reduced the attributes to 26 selected attributes. Now the goal was to compare the reduced dataset with the baseline experiment result. We used the same five classification algorithms to construct the model, namely NaiveBayes, SMO, ZeroR, J48 and RandomForest.

5) Evaluation: Comparing the accuracy results from this experiment and the fourth experiment was reported in Table 9 and Table 10. As a result, there was no enhancement in general. Only J48 1.38\%. 
Table 9: Experiment 5 confusion matrix results.

\begin{tabular}{|c|c|c|c|c|c|}
\hline \multirow[b]{2}{*}{ Algorithm } & \multirow[b]{2}{*}{ Learning Machine } & \multicolumn{4}{|c|}{ Detailed Accuracy } \\
\hline & & 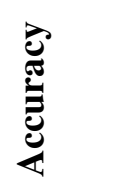 & 总 & 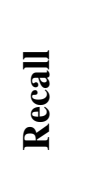 & 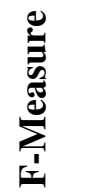 \\
\hline Bayes & NavieBayes & $73.17 \%$ & 0.709 & 0.732 & 0.702 \\
\hline Functions & SMO & $73.68 \%$ & 0.726 & 0.737 & 0.684 \\
\hline Rule & ZeroR & $70.24 \%$ & 0.493 & 0.702 & 0.580 \\
\hline Tree & $\mathrm{J} 48$ & $73.82 \%$ & 0.726 & 0.738 & 0.730 \\
\hline Tree & RandomForest & $74.65 \%$ & 0.731 & 0.747 & 0.733 \\
\hline
\end{tabular}

Table 10: Accuracy comparison between Experiment 4 \& Experiment 5.

\begin{tabular}{|l|l|l|l|r|}
\hline Algorithm & $\begin{array}{c}\text { Learning } \\
\text { Machine }\end{array}$ & $\begin{array}{c}\text { Accuracy of } \\
\text { the original } \\
\text { total number } \\
\text { of tests } \\
\text { dataset }\end{array}$ & $\begin{array}{c}\text { Accuracy of the } \\
\text { reduced total } \\
\text { number of tests } \\
\text { dataset }\end{array}$ & Change \\
\hline Bayes & NavieBayes & $73.48 \%$ & $73.17 \%$ & $-0.31 \%$ \\
\hline Functions & SMO & $74.85 \%$ & $73.68 \%$ & $-1.17 \%$ \\
\hline Rule & ZeroR & $69.72 \%$ & $70.24 \%$ & $0.52 \%$ \\
\hline Tree & J48 & $72.44 \%$ & $73.82 \%$ & $1.38 \%$ \\
\hline Tree & RandomForest & $75.30 \%$ & $74.65 \%$ & $-0.65 \%$ \\
\hline
\end{tabular}

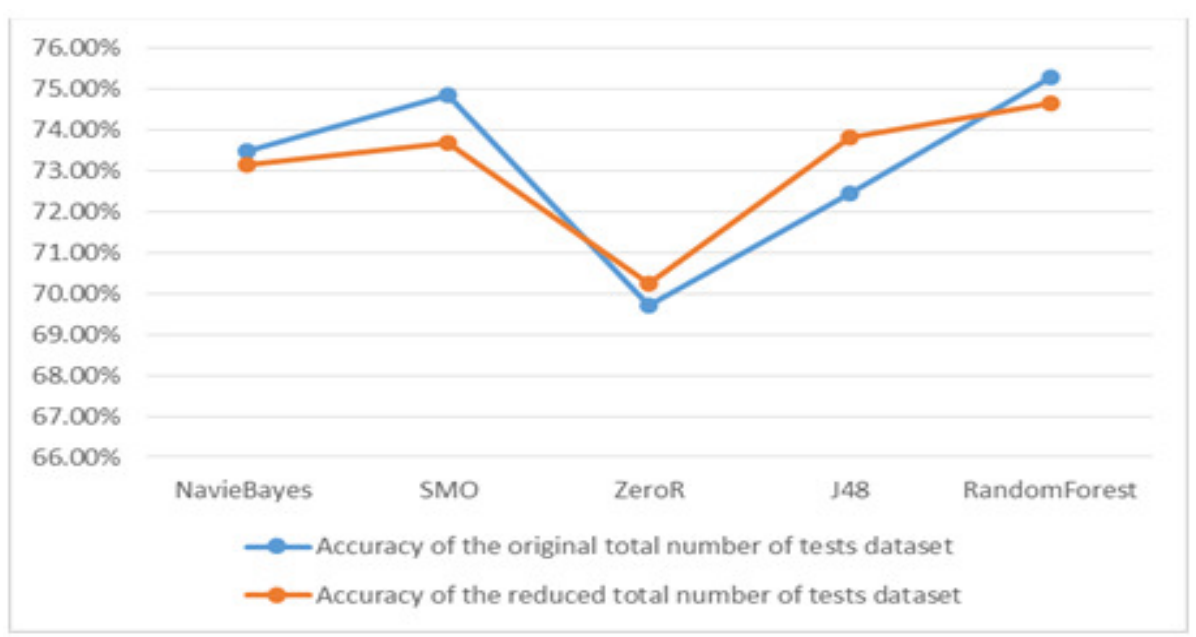

Figure 8: Accuracy comparison between Experiment 4 \& Experiment 5.

\subsection{Experiment 6: Feature Selection for Total Number of Medical Lab Tests}

1) Experiment Goal: The goal of this experiment was to study the relationship between feature selection and classification accuracy. Feature selection is one of the dimensionality reduction 
International Journal of Data Mining \& Knowledge Management Process (IJDKP) Vol.5, No.6, November 2015

techniques for reducing the attribute space of a feature set. More precisely, it measures how many features should be enough to give moderate accuracy.

2) Building the Dataset: In this experiment we used a count dataset.

3) Pre-processing: In the pre-processing step, we built ten datasets depending on the number of selected features. The first dataset contained only $10 \%$ of the total attributes. Then we increased the total feature selections by $10 \%$ with each new dataset. For example, dataset 1 contained $10 \%$ of the total attributes, dataset 2 contained $20 \%$ of the total attributes, dataset 3 contained $30 \%$ of the total attributes and so on till dataset 10 contained all $100 \%$ of the total attributes.

4) For feature selection, we used supervised.attribute. InfoGainAttributeEval from WEKA. This filter is a wrapper for the Weka class that computes the information gain on a class [20].

- Attribute Subset Evaluator: InfoGainAttributeEval

- Search Method: Ranker.

- Evaluation mode: evaluate on all training data

5) Base learner: After generating all reduced datasets, we used the J48 algorithm as a base learner.

Table 11: Experiment 4 Results.

\begin{tabular}{|c|c|c|c|c|}
\hline \multirow[b]{2}{*}{$\%$ of Features Selection } & \multirow[b]{2}{*}{ \# of Features Selection } & \multicolumn{3}{|c|}{ Detailed Accuracy } \\
\hline & & 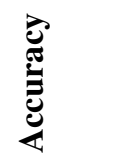 & 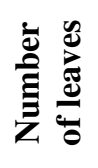 & 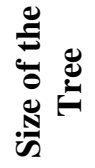 \\
\hline $10 \%$ & 62 & $71.45 \%$ & 237 & 473 \\
\hline $20 \%$ & 124 & $73.90 \%$ & 250 & 499 \\
\hline $30 \%$ & 186 & $73.55 \%$ & 247 & 493 \\
\hline $40 \%$ & 248 & $72.79 \%$ & 252 & 503 \\
\hline $50 \%$ & 310 & $73.41 \%$ & 252 & 503 \\
\hline $60 \%$ & 371 & $73.66 \%$ & 254 & 507 \\
\hline $70 \%$ & 433 & $74.24 \%$ & 254 & 507 \\
\hline $80 \%$ & 495 & $74.10 \%$ & 254 & 507 \\
\hline $90 \%$ & 557 & $74.14 \%$ & 265 & 529 \\
\hline $100 \%$ & 619 & $73.59 \%$ & 259 & 517 \\
\hline
\end{tabular}

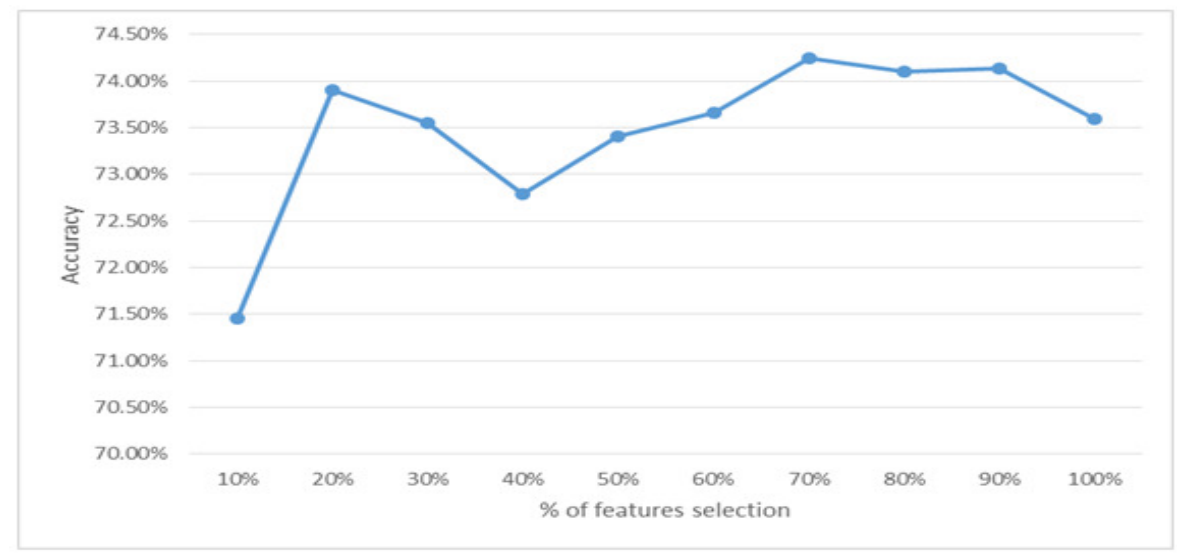

Figure 7: Count Dataset accuracy. 
6) Evaluation: Each feature-reduced dataset went through a 10-fold cross-validation for evaluation. Figure 9 shows the accuracy of all count datasets. The detail values are also reported in Table 9. From the results we observe that selecting 60 to $70 \%$ of the attributes gives the highest accuracy. This also concludes that all features (i.e., lab tests) may not be necessary to attain a highly accurate prediction of patient deterioration.

\section{DISCUSSION}

In the experiment we investigated the effect of feature selection in improving the prediction of patient deterioration in the ICUs. We considered the lab tests as features. Thus, choosing a subset of features would mean choosing the most important lab tests to perform. If the number of tests could be reduced by identifying the most important tests, then we would also identify the redundant tests. It should be noted that the feature selections were done without any domain knowledge and without any intervention from medical experts. However, in the analysis we would like to emphasize the merit of feature selection in choosing the best tests, which could be further verified and confirmed by a medical expert.

First we compare the selected features selected from the two datasets, namely the average dataset and the count dataset. Table 12 shows the 10 best features chosen by the two approaches and highlights the common lab tests between the two approaches (i.e. using the average of tests and count of tests). Table 13 shows more details about the common tests.

Table 11: Final Results.

\begin{tabular}{|l|l|l|}
\hline & Average Dataset & Count Dataset \\
\hline & 50177 & 50148 \\
& 50090 & 50112 \\
& 50060 & 50140 \\
Best ranked 10 from the 10\% of selected features & 50399 & 50399 \\
& 50386 & 50177 \\
& 50440 & 50439 \\
& 50408 & 50090 \\
& 50439 & 50440 \\
& 50112 & 50079 \\
\hline
\end{tabular}


Table 13: Medical Lab Test Details.

\begin{tabular}{|c|c|c|c|c|c|}
\hline & \multicolumn{5}{|c|}{ Detailed Description } \\
\hline & 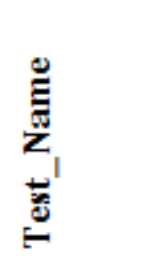 & 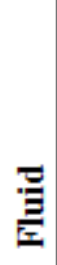 & 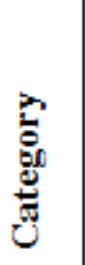 & 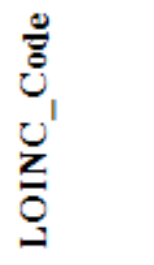 & 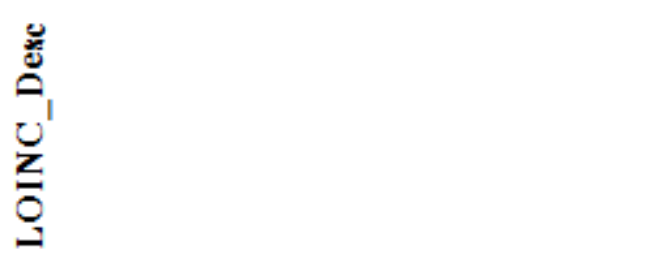 \\
\hline 50177 & UREA N & $\begin{array}{c}9 \\
9 \\
9 \\
\end{array}$ & 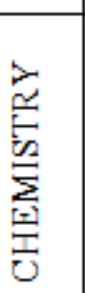 & $3094-0$ & $\begin{array}{l}\text { Urea nitrogen [mass/volume] in serum or } \\
\text { plasma }\end{array}$ \\
\hline 50090 & CREAT & $\stackrel{\ominus}{9}$ & 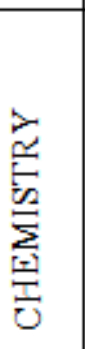 & $2160-0$ & $\begin{array}{l}\text { Creatinine [mass/volume] in serum or } \\
\text { plasma }\end{array}$ \\
\hline 50399 & $\overline{\text { INR(PT) }}$ & $\stackrel{\ominus}{\bigcirc}$ & 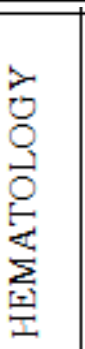 & $\overline{34714-6}$ & INR in blood by coagulation assay \\
\hline 50440 & PTT & 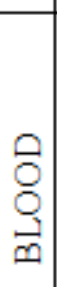 & 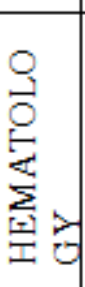 & $3173-2$ & $\begin{array}{l}\text { Activated partial thromboplastin time } \\
\text { (aPTT) in blood by coagulation assay }\end{array}$ \\
\hline 50439 & PT & $\stackrel{\rho}{9}$ & 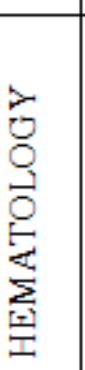 & $5964-2$ & $\begin{array}{l}\text { Prothrombin time (PT) in blood by } \\
\text { coagulation assay }\end{array}$ \\
\hline
\end{tabular}


International Journal of Data Mining \& Knowledge Management Process (IJDKP) Vol.5, No.6, November 2015

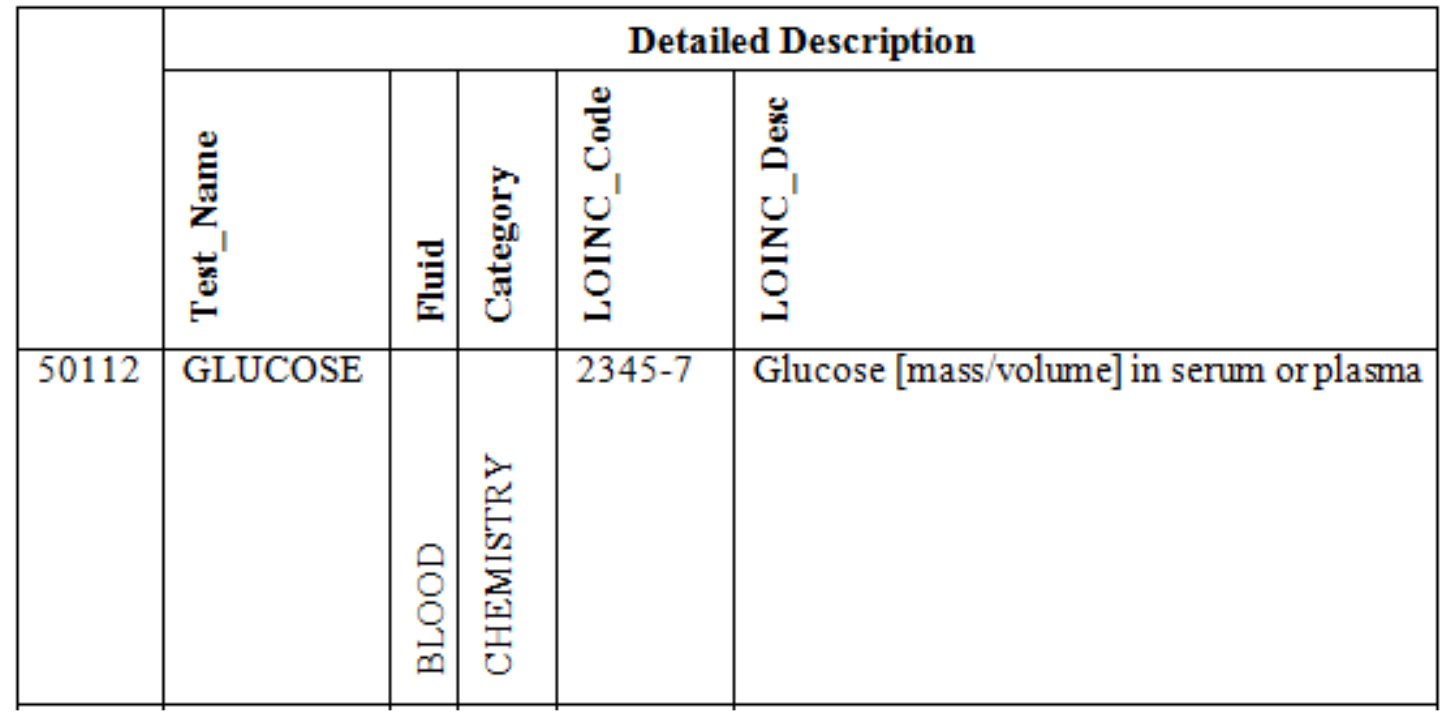

LOINC is an abbreviation for logical observation identifiers names and codes. LOINC is clinical terminology important for laboratory test orders and results [21]. ARUP Laboratories [22] is a national clinical and anatomic pathology reference laboratory and a worldwide leader in innovative laboratory research and development. We used their web page and others to clarify more about the medical lab tests in table 10 as follows:

- UREAN (50177): This test is conducted using the patient's blood. This test is recommended to screen for kidney dysfunction in patients with known risk factors (e.g. hypertension, diabetes, obesity, family history of kidney disease). The panel includes albumin, calcium, carbon dioxide, creatinine, chloride, glucose, phosphorous, potassium, sodium and BUN and a calculated anion gap value. Usually, the result is reported within 24 hours [22].

- CREAT (50090): This test is conducted using the patient's blood. It is a screening test to evaluate kidney function [22].

- INR(PT) (50399): This test is conducted using the patient's blood by coagulation assay [15].

- PTT (50440): This test is carried out to answer two main questions: does the patient have antiphospholipid syndrome (APLS), and does the patient have von Willebrand disease? If so, which type? It is carried out by mechanical clot detection [23].

- PT (50439): This test is conducted using the patient's blood by coagulation assay [15].

- GLUCOSE (50112): This test is used to check glucose, which is a common medical analytic measured in blood samples. Eating or fasting prior to taking a blood sample has an effect on the result. Higher than usual glucose levels may be a sign of prediabetes or diabetes mellitus [24].

- The result of the top 10 selected features from the average dataset allows us to build a model using decision tree $\mathrm{J} 48$. This model would allow a medical professional to predict the status of a patient in the ICU as follows: 


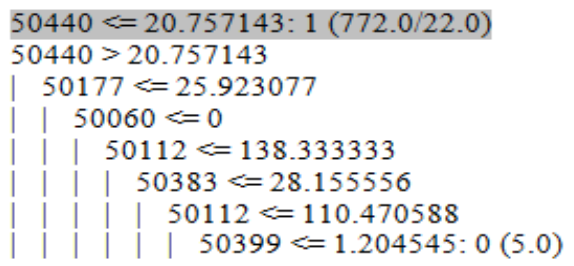

For example, if the lab test (name: PTT, ID 50440, LOINC: 3173-2) result value is $<=$ 20.757143 , then the probability is very high $(772.0 / 22.0 \sim 97.2 \%)$ that the patient is going to die (class:1). This model has $78.6897 \%$ overall accuracy.

\section{CONCLUSION AND FUTURE WORK}

The increasing amount of medical laboratory data represents a significant information resource that can provide a foundation for improved understanding of patients' critical. Data mining supports this goal by providing a set of techniques designed to discover similarities and relationships between data elements in large data sets.

Reducing frequent laboratory testing and the potential care and financial implications are critical issues in the intensive care units. In this paper, we presented our proposed approach to reduce the observation time in the ICU by predicting patient deterioration in its early stages. In our work, we presented six experiments to investigate the effect of the average laboratory test value and number of total laboratory in predicting patient deterioration in the Intensive Care Unit. In our work, we considered laboratory tests as features. Choosing a subset of features would mean choosing the most important lab tests to perform.

For future work, the authors are planning to carry out more experiments using bigger data. Big data analytics would bring potential benefits to support taking the right decision to enhance the efficiency, accuracy and timeliness of clinical decision making in the ICU.

\section{REFERENCES}

[1] Federico Cismondi, Leo A. Celi, André S. Fialho, Susana M. Vieira, Shane R. Reti, Joao MC Sousa, and Stan N. Finkelstein, "Reducing unnecessary lab testing in the ICU with artificial intelligence," Int. J. Med. Inf., vol. 82, no. 5, pp. 345-358, 2013.

[2] Yi Mao, Wenlin Chen, Yixin Chen, Chenyang Lu, Marin Kollef, and Thomas Bailey, "An integrated data mining approach to real-time clinical monitoring and deterioration warning," in Knowledge discovery and data mining, 2012, pp. 1140-1148.

[3] Masha Rouzbahman and Mark Chignell, "Predicting ICU Death with Summarized Data: The Emerging Health Data Search Engine.," KMD, 2014.

[4] Q. Liu, Sung, Andrew H, Ribeiro, Bernardete, and Suryakumar, Divya, "Mining the Big Data: The Critical Feature Dimension Problem,” Adv. Appl. Inform. IIAIAAI 2014 IIAI 3rd Int. Conf. On, pp. 499-504, 2014.

[5] Joon Lee and David M. Maslove, "Using information theory to identify redundancy in common laboratory tests in the intensive care unit," BMC Med. Inform. Decis. Mak., vol. 15, no. 1, 2015.

[6] Yi-Zeng Hsieha, Mu-Chun Sua, Chen-Hsu Wangb, and Pa-Chun Wangc, "Prediction of survival of ICU patients using computational intelligence," Comput. Biol. Med., vol. 47, pp. 13-19, 2014.

[7] J. Iavindrasana, G. Cohen, A. Depeursinge, H. Müller, R. Meyer, and A. Geissbuhler, "Clinical data mining: a review," Yearb Med Inf., pp. 121-133, 2009.

[8] Yvan Saeys, Iñaki Inza, and Pedro Larrañaga, "A review of feature selection techniques in bioinformatics," bioinformatics, vol. 23, no. 19, pp. 2507-2517, 2007.

[9] "An Introduction to Feature Selection - Machine Learning Mastery." [Online]. Available: http://machinelearningmastery.com/an-introduction-to-feature-selection/. [Accessed: 06-Sep-2015]. 
International Journal of Data Mining \& Knowledge Management Process (IJDKP) Vol.5, No.6, November 2015

[10] S. Bouktif et al, "Ant Colony Optimization Algorithm for Interpretable Bayesian Classifiers Combination: Application to Medical Predictions," PLoS ONE, vol. 9, no. 2, 2014.

[11] X. Wu et al., "Top 10 algorithms in data mining," Knowl. Inf. Syst., vol. 14, no. 1, pp. 1-37, 2008.

[12] Chitra Nasa and Suman, "Evaluation of Different Classification Techniques for WEB Data," Int. J. Comput. Appl., vol. 52, no. 9, 2012.

[13] John C. Platt, "Sequential Minimal Optimization: A Fast Algorithm for Training Support Vector Machines," Adv. Kernel Methods—support Vector Learn., vol. 3, 1999.

[14] Leo Breiman, "Random Forests,” Mach. Learn., vol. 45, no. 1, pp. 5-32, 2001.

[15] "MIMIC II Database." [Online]. Available: https://mimic.physionet.org/database.html. [Accessed: 20-Aug2015].

[16] Lee J, Govindan S, Celi L, Khabbaz K, and Subramaniam B, "Customized prediction of short length of stay following elective cardiac surgery in elderly patients using a genetic algorithm," World J Cardiovasc Surg, vol. 3, no. 5, pp. 163-170, Sep. 2013.

[17] Lehman LH, Saeed M, Talmor D, Mark R, and Malhotra A, "Methods of blood pressure measurement in the ICU," Crit Care Med, vol. 41, no. 1, pp. 34-40, 2013.

[18] Lehman L, Long W, Saeed M, and Mark R, "Latent topic discovery of clinical concepts from hospital discharge summaries of a heterogeneous patient cohort," in Proceedings of the 36th International Conference of the IEEE Engineering in Medicine and Biology Society, 2014.

[19] "Laboratory Test Reference Ranges I Calgary Laboratory Services." [Online]. Available: https://www.calgarylabservices.com/lab-services-guide/lab-reference-ranges/. [Accessed: 03-Sep-2015].

[20] "Feature Selection Package Documentation." [Online]. Available: http://featureselection.asu.edu/documentation/infogain.htm. [Accessed: 04-Sep-2015].

[21] “LOINC Codes - Mayo Medical Laboratories." [Online]. Available: http://www.mayomedicallaboratories.com/test-catalog/appendix/loinc-codes.html. [Accessed: 10-Sep-2015].

[22] “ARUP Laboratories: A National Reference Laboratory.” [Online]. Available: http://www.aruplab.com/. [Accessed: 10-Sep-2015].

[23] "UCSF Departments of Pathology and Laboratory Medicine I Lab Manual I Laboratory Test Database I Activated Partial Thromboplastin Time." [Online]. Available: http://labmed.ucsf.edu/labmanual/db/data/tests/802.html. [Accessed: 10-Sep-2015].

[24] “2345-7.” [Online]. Available: http://s.details.loinc.org/LOINC/2345-7.html?sections=Comprehensive. [Accessed: 10-Sep-2015].

\section{AUTHORS}

Noura Al Nuaimi is pursuing a PhD in Information Technology with Dr Mohammad Mehedy Masud at United Arab Emirates University (UAEU). She holds an MSc in Business Administration from Abu Dhabi University and a BSc in Software Engineering from UAEU. Her research interests focus on data mining and knowledge discovery, cloud computing, health information systems, search engines and natural language processing. She has published research papers in IEEE Computer Society and IEEE Xplore.

Dr Mohammad Mehedy Masud is currently an Assistant Professor at the United Arab Emirates University (UAEU). He joined the College of Information Technology at UAEU in spring 2012. He received his $\mathrm{PhD}$ from University of Texas at Dallas (UTD) in December 2009. His research interests are in data mining, especially data stream mining and big data mining. He has published more than 30 research papers in journals including IEEE Transactions on Knowledge and Data Engineering (TKDE), Journal of Knowledge and Information Systems (KAIS), ACM Transactions on Management Information Systems (ACM TMIS) and peer-reviewed conferences including IEEE International Conference on Data Mining (ICDM), European Conference on Machine Learning (ECML/PKDD) and Pacific Asia Conference on KDD. He is the principal inventor of a US patent application and lead author of the book "Data Mining Tools for Malware Detection". Dr Masud has served as a program committee member of several prestigious conferences and has been serving as the official reviewer of several journals, including IEEE TKDE, IEEE TNNLS and DMKD. During his service at the UAEU he has secured several internal and external grants as PI and co-PI.

Farhan Mohammed is a graduate from the College of Information Technology in United Arab Emirates University specializing in Information Technology Management. He obtained his Bachelor's in Management Information Systems from United Arab Emirates University, Al Ain, UAE. He has worked under several professors and published four conference papers and a journal paper for IEEE sponsored conferences. Currently he is working as a research assistant in data mining in the health industry to develop models on health deterioration prediction. His area of interests lies in smart cities, UAVs, data mining, and image and pattern recognition. 